\title{
EL DESARROLLO DE TECNOLOGÍAS PARA LA GESTIÓN DEL PATRIMONIO ARQUEOLÓGICO: HACIA UN MODELO DE EVALUACIÓN DEL IMPACTO ARQUEOLÓGICO
}

\author{
DEVELOPING TECHNOLOGIES \\ FOR THE MANAGEMENT OF THE ARCHAEOLOGICAL \\ HERITAGE: TOWARDS A MODEL OF EVALUATION \\ OF THE ARCHAEOLOGICAL IMPACT
}

\section{DAVID BARREIRO MARTÍNEZ (*) VICTORIA VILLOCH VÁZQUEZ $(*)$ FELIPE CRIADO BOADO $(*)$}

"Quien por distracción o incompetencia detenga, aunque sólo sea un momento, la marcha de la humanidad, será su salvador".

E. M. Cioran: Silogismos de la amargura.

\section{RESUMEN}

Este texto plantea la necesidad de establecer procedimientos y convenciones de práctica arqueológica para resolver los problemas y demandas en la gestión actual del Patrimonio Arqueológico. En concreto se presenta el programa CAPA (criterios y convenciones en Arqueología del Paisaje y Patrimonio Arqueológico) en vía de realización por nuestro Grupo de Investigación. El objetivo de este programa es contribuir al desarrollo de sistemas y metodologías de gestión y evaluación del PatrimonioArqueológico y llegar a componer un Manual de Usos Internos que nuestro grupo aplicaría en los diferentes proyectos de investigación y de asistencia técnica. Como ejemplo de estos desarrollos, se considera la Evaluación de Impacto Arqueológico y se propone un esquema metodológico para la realización de este nuevo tipo de práctica arqueológica, que forma parte importante de la Evaluación de Impacto Ambiental.

(*) Grupo de investigación en Arqueología del Paisaje, Universidade de Santiago de Compostela. Laboratorio de Arqueología. Edificio Monte da Condesa USC - Campus Sur. 15706 Santiago de Compostela. España. Correo electrónico: pharpa@usc.es.

El artículo fue remitido en su versión final el 2-IX-98.

\begin{abstract}
This paper proposes to establish criteria and procedures of archaeological practice to solve the problems and demands posed by the Management of the Archaeological Heritage. OurResearch Unit is developing the CLAAR Programme, to define criteria and conventions for Landscape Archaeology and Archaeological Heritage. The main aim of this programme is to contribute to the development of systems and methodologies to manage and evaluate the Archaeological Heritage. In a practical sense, it tries to compose a Handbook of Practice to apply to research and commercial projects. As an example, this paper deals with the Evaluation of Archaeological Impact, proposing a methodological scheme to be applied to this new practice, which is an important part of the Evaluation of Environmental Impact.
\end{abstract}

Palabras clave: PatrimonioArqueológico. Gestión del PatrimonioArqueológico. Evaluación de ImpactoAmbiental. Evaluación de Impacto Arqueológico. Arqueología del Paisaje.

Key words: Archaeological Heritage. Management of Archaeological Heritage. Environmental Assessment. Archaeological Impact Assessment. Landscape Archaeology. 


\section{LA INVESTIGACIÓN APLICADA: NUEVAS FRONTERAS PARA LA ARQUEOLOGÍA}

El desarrollo de la gestión del Patrimonio Arqueológico como campo especializado de actividad genera necesidades prácticas inéditas. La disciplina arqueológica debe responder a éstas adaptando sus metodologías, criterios e, incluso, objetivos de trabajo. En cierto sentido, la Arqueología tiene que empezar a considerar que su finalidad única y prioritaria ya no es excavar yacimientos ni puede ser reconstruir el pasado arqueológico en abstracto. Sino que, parte al menos de sus objetivos prácticos, se deben encardinar con la gestión de la problemática presentada por el Patrimonio Arqueológico en el contexto de una sociedad compleja y plural.

Esto implica que la Arqueología debe desarrollar, establecer y consensuar formas de trabajo en contextos innovadores, determinados por temas y ámbitos relacionados con la gestión del Patrimonio Arqueológico (PArq en adelante). Conviene concretar en qué sentido se utiliza el término gestión, dado que se tiende a vincularlo de forma directa y exclusiva con laAdministración, lo que no sólo es erróneo sino que además levanta suspicacias. Por nuestra parte entendemos gestionar como: aplicar conocimiento y administrar recursos (generalmente escasos) para resolver problemas concretos que, lejos de ser abstractos, están vinculados a condiciones objetivas específicas.

Ahora bien, para reconvertir la Arqueología en una disciplina de gestión del PArq es necesario ante todo apurar el desarrollo de metodologías, procedimientos y especificaciones, entendiendo estas últimas como la definición de directrices sobre qué y cómo hacer para gestionar ese Patrimonio. Simultáneamente es preciso establecer criterios tanto de evaluación como de intervención en todos los nuevos frentes de acción vinculados a esta gestión. Y por último es necesario alcanzar un elevado grado de consenso sobre estos desarrollos dentro de la disciplina y con los agentes sociales implicados. Esto supone un proceso de debate público, que puede tener lugar por los medios habituales para esta finalidad, empezando por la correcta difusión de los avances en criterios y especificaciones.

Pero antes hay que acrecentar estos avances, incorporando laArqueología a la política de I+D (innovación y desarrollo tecnológico). La tecnología debería dejar de ser concebida como algo ajeno a las disciplinas humanísticas. En cambio hay que con- siderarla como lo que es, un saber-hacer o saber teórico que permite hacer cosas, para incorporar así nuestra disciplina al desarrollo tecnológico y a la investigación aplicada, centrándonos en nuestro caso en la puesta a punto de procedimientos y valores prácticos para resolver los problemas que presenta la existencia del PArq y reconvertir éste en recursos culturales que puedan ser adecuadamente rentabilizados por la sociedad (Criado, 1996a: 17).

En este contexto, el Grupo de Investigación en Arqueología del Paisaje de la Universidad de Santiago de Compostela ha puesto en marcha el Programa CAPA, acrónimo de Criterios y Convenciones en Arqueología del Paisaje y Patrimonio Arqueológico. Es un programa de trabajo propio del grupo (es decir, no subvencionado por ningún proyecto de investigación o institución concreta) que atraviesa verticalmente diferentes proyectos y líneas de trabajo en curso de realización, y que constituye un programa de investigación aplicada y de desarrollo tecnológico en el sentido antes apuntado. El objetivo que persigue es innovar en metodología arqueológica y, sobre todo, adaptar ésta a las nuevas demandas prácticas citadas, mediante la retroalimentación con los trabajos concretos y aprovechándose de las soluciones específicas que se ponen coyunturalmente a punto para solventar los problemas prácticos que la ejecución de todo proyecto supone.

El Programa CAPA pretende contribuir a que los trabajos más tradicionales de investigación básica, centrados en la interpretación del registro arqueológico, se puedan completar en Arqueología con una investigación aplicada al desarrollo de metodologías de Gestión y Evaluación del Patrimonio Cultural. Consideramos urgente perfeccionar esta temática porque la consolidación de esta actividad y del mercado de trabajo a ella asociado depende en gran medida de la capacidad de laArqueología para responder a problemas prácticos, hacerlo con agilidad y eficacia y de un modo que sea objetivo o, cuando menos, fiable. Lo que está en juego por lo tanto es una plena profesionalización del trabajo arqueológico, entendiendo por ello no lo que habitualmente se entiende en nuestro país (a saber, que ese trabajo lo realicen profesionales), sino que la Arqueología se desarrolle para intervenir de forma ágil y productiva en los diferentes ámbitos del estudio y gestión del PArq y que de esta forma crezca un sector profesional maduro y capaz.

El Programa CAPA tiene por objetivo concreto producir una serie de especificaciones técnicas que 
contengan presentaciones sintéticas de los criterios, procedimientos y convenciones de actuación en diferentes frentes. Constituye en su conjunto una suerte de manual interno de usos y estándares de nuestro grupo de investigación, que permite mantener formas de trabajo homogéneas, lo que es básico dentro de un equipo numeroso y de una dinámica que es, fundamentalmente, interactiva (diferentes instancias e individuos intervienen en diferentes momentos de un mismo proyecto) e iterativa (se vuelve en momentos sucesivos sobre las mismas circunstancias y entidades de información). Asimismo, facilita la integración y formación de personal colaborador que se incorpora a los diferentes proyectos.

Para ello, el manual CAPA debía ser accesible. Esta necesidad se cubrió incluyendo los diferentes documentos con especificaciones técnicas en la Intranet del grupo. En la actualidad se dispone de 344 páginas con este material, que están elaboradas con tecnología hipertextual, lo que permite acceder con facilidad a los segmentos de información de interés concreto. El índice general de esta sección de la Intranet se puede observar en la tabla 1 (1).

Dado que el conjunto de este material no está totalmente verificado y que su utilidad es en muchos casos fundamentalmente interna, el manual CAPA no es de acceso público generalizado. Recientemente, sin embargo, pusimos en marcha una iniciativa editorial para publicar y difundir aquella parte de este material que cumpla la doble condición de ser de amplia utilidad y haber sido verificado en periodos de aplicación práctica más o menos largos. De este modo, el Programa CAPA se concreta en la serie de cuadernos $C_{A P A}$, que presentan los criterios, convenciones, procedimientos y técnicas de trabajo utilizados por nosotros (2).

Como ejemplificación del Programa CAPA se ha optado por mostrar de forma sumaria el desarrollo de la metodología arqueológica necesaria para operar en un campo nuevo como es la Evaluación de Impacto Ambiental (EIA en adelante), que implica establecer criterios y procedimientos de Evaluación de ImpactoArqueológico (EIArq en adelante) y concretar los mismos en forma de espe-

(1) El diseño y mantenimiento de este servicio es responsabilidad de la unidad de telemática del grupo, concretamente de su coordinador (C. González) y del administrador de sistemas (R. Gómez).

(2) Los títulos publicados de la serie CAPA aparecen en la bibliografía. Se distribuyen en versión impresa o a través de Internet (http://www-gtarpa.usc.es/CAPA, y http://www-gtarpa.usc.es/ TAPA para la serie de Trabajos en Arqueología del Paisaje).
Teoría y Cuestiones Generales

1. Arqueología y Registro Arqueológico

2. Modelos de Gestión: la Práctica Arqueológica como Cadena Valorativa

3. Definición, Objetivos y Dimensiones de la $\mathrm{ArPa}$

4. Definición de una Matriz Teórico-Metodológica para la ArPa

5. Procedimientos Analíticos en ArPa 1. Análisis de Terrenos

\section{Conceptos y Vocabulario}

1. Conceptos de Arqueología y Arqueología del Paisaje

2. Conceptos Técnicos en Gestión y Evaluación de PArq 1. Conceptos en Valoración

2. Conceptos en Evaluación de Impacto

3. Conceptos en Corrección de Impacto

4. Conceptos de Cultura Material

3. Vocabulario de Términos Organizativos y de Gestión

4. Vocabulario Divulgativo de Arqueología y PArq

5. Conceptos de Geografía

1. Conceptos de Geografía Física

2. Conceptos de Geografía Humana

6. Glosario general

\section{Metodología}

1. Sistemas de Inventario

1. Inventario Arqueológico

1. Objetivos de Inventario

1. Categorización de Yacimientos Arqueológicos en Galicia

2. Metodología

1. Sistemas de Prospección

3. Definición de Condiciones de Entorno y Emplazamiento

2. Inventario Construido o «Emergente»

3. Inventario Etnográfico

2. Gestión del Inventario y Ámbitos de Actuación

1. Valoración del Patrimonio Arqueológico

2. Planeamiento

3. Impacto Arqueológico

1. Evaluación del Impacto

2. Corrección del Impacto

4. Rentabilización

3. Excavación

1. Registro de Información

2. Manipulación y Análisis de Muestras

4. Gestión y Diagnóstico de Cultura Material

\section{Organización}

1. Gestión de Proyectos

. Bibliografía

3. Manual de Estilo

\section{Sistemas de Archivo}

1. Gestión de Informes

1. Tipos y Nomenclatura de Informes

2. Fotografía

3. Videografía

4. Dibujos

5. Planimetría y Mapas

6. Diario

7. Archivo Documental

8. Planillas de Campo

Tab. 1. Contenido del Programa CAPA. 
cificaciones para la realización de esta práctica arqueológica (3).

Estas especificaciones han surgido al hilo de los estudios de EIArq que nuestro grupo realizó en diferentes contextos (Criado, 1995; Criado et alii, 1995; González et alii, 1995; Méndez et alii, 1995a; Méndez et alii, 1995b; Criado et alii, 1997 y Villoch y Barreiro, 1997). La necesidad de hacer estos trabajos había sido adelantada por los responsables de los Servicios de Arqueoloxía de la Consellería de Cultura gallega, que han insistido en la importancia de realizar evaluaciones de impacto y en la inevitabilidad de llevar a cabo seguimientos arqueológicos de los proyectos de construcción (Tallón, 1993).

Todas estas actuaciones han posibilitado predefinir un esquema metodológico para evaluar y corregir el Impacto Arqueológico de la construcción de Obras Públicas (4). Paulatinamente se ha podido definir tanto el propio concepto de Impacto Arqueológico, como el de Evaluación de ImpactoArqueológico, y observar su articulación con la Evaluación de Impacto Ambiental, entendida ésta como el "proceso de análisis (...) encaminado a formar un juicio previo, lo más objetivo posible, sobre los efectos ambientales de una acción humana prevista (...) y sobre la posibilidad de evitarlos o reducirlos a niveles aceptables" (Gómez, 1994: 35).

A este respecto, la normativa existente (comunitaria, estatal y autonómica) considera el Patrimonio Cultural, incluyendo el Arqueológico, como uno de los factores que conforman el MedioAmbiente. Sin embargo, las principales guías metodológicas para la EIA tratan el tema de un modo tangencial y, en algunos casos, anecdótico, lo cual no deja de ser lógico, teniendo en cuenta la singularidad de la Arqueología respecto a otras disciplinas manejadas en los estudios de impacto ambiental (5). Nuestro primer objetivo es, por lo tanto, el desarrollo de una metodología que permita la inclusión del PArq como un factor específico dentro del proceso global de EIA, y orientada igual que ésta por parámetros no sólo cualitativos sino también cuantitativos.

(3) Este texto se completa con otro en preparación por parte de F. Criado, J. Amado y M.C. Martínez en el que se continúa la temática aquí tratada, relativa a la EIArq antes de las obras, con el análisis y definición de las estrategias de Corrección del Impacto durante la fase de construcción.

(4) Las bases metodológicas de este trabajo han sido definidas gracias al esfuerzo, desde 1991, de todos los componentes de nuestro Grupo de Investigación. Nuestro agradecimiento a todos ellos.

(5) Véase Gómez, 1994; Conesa, 1995, o cualquiera de las Guías editadas sobre el tema por el Ministerio de Obras Públicas y Transportes.
Desde un punto de vista conceptual, podemos entender por EIArq el proceso de análisis por el que se identifica (relaciones causa-efecto), predice (diagnosis y previsión de impactos), valora (evaluación), previene (introducción de medidas correctoras) y comunica (participación pública) el impacto de un proyecto sobre el PArq (Gómez, 1988). Este proceso se articula, sintéticamente, en cuatro fases: el análisis del proyecto, la elaboración del inventario de bienes arqueológicos que pueden ser afectados, la evaluación de esa afección (que constituye la EIArq propiamente dicha) y la definición de las medidas correctoras de ese impacto. Estas fases no deben ser consideradas como eslabones fijos dentro de la cadena valorativa, sino como estadios intermedios de un proceso retroalimentado, como se podrá observar en cada uno de los apartados de este texto, a medida que se expongan los criterios y procedimientos que los orientan.

\section{ANÁlISIS DEL PROYECTO DE OBRA}

Siguiendo el orden establecido más arriba, el proceso de EIArq debe comenzar por un análisis en profundidad del proyecto que se somete a evaluación. Se requieren para ello una serie de pautas (6) que permitan describir y comprender las proporciones y características del proyecto, definir los componentes del mismo para identificar aquellas acciones que pueden producir afecciones sobre el PArq y delimitar el ámbito geográfico afectado (7).

En primer lugar se debe establecer el tipo de proyecto de que se trata. Es preciso especificar, por lo tanto, el nivel de articulación administrativa en el que se enmarca: si se trata de una actuación puntual y determinada, con lo que el análisis se constriñe a un espacio definido y localizado, o bien si pertenece a un plan o programa sectorial (como podría ser el caso del Plan Eólico de Galicia), con lo que es factible un análisis genérico y válido para más de una actuación.

En segundo lugar, la descripción general del proyecto debe determinar la situación gerencialen la que éste se encuentra en el momento del estudio de IArq. Para ello se sigue un programa de desarro-

(6) Para ello es preciso seguir, readaptándolas, las directrices emanadas del Reglamento para la ejecución del Real Decreto Legislativo 1302/86, en su artículo $8^{\circ}$ (Descripción del proyecto y sus acciones. Examen de alternativas), desarrollado en el Real Decreto $1131 / 88$, de 30 de septiembre.

(7) Se puede encontrar un ejemplo exhaustivo de análisis en Barreiro y Villoch, 1997.

T. P., 56, n. ${ }^{\circ} 1,1999$ 
llo, general para todo tipo de proyectos que impliquen una modificación física del espacio, que distingue entre las fases de planificación, construcción, explotación, previsiones de modificación y, eventualmente, abandono o desmantelamiento. Para un correcto proceso de evaluación, el momento idóneo para el estudio es la fase de planificación. De no ser así, la minimización de los efectos sobre el PArq resultará más compleja, pues será difícil elaborar previsiones y proponer medidas correctoras. Igualmente, será necesario determinar las características específicas del proyecto, especialmente en cuanto a exigencias de uso del suelo, para delimitar con mayor precisión las áreas de afección e incidencia (conceptos definidos más abajo), y a la tecnología que se empleará en las diferentes fases (lo que permitirá precisar las acciones susceptibles de producir efectos sobre el medio).

En un nivel más concreto, y basándonos en los resultados obtenidos en esta primera aproximación, el análisis de los distintos componentes que conforman el proyecto, es decir, cada uno de los elementos que lo integran, considerados desde el punto de vista de su potencialidad para producir impactos ambientales o arqueológicos, nos permitirá identificar con precisión y desagregar tres niveles diferentes: agentes, factores y acciones.

Por agente entendemos aquel componente del proyecto, físico y concreto, a causa del cual tiene lugar una afección. Se consideran agentes todas las infraestructuras e instalaciones necesarias para la construcción y el funcionamiento del proyecto, desde las obras de instalación específicas que configuran su singularidad hasta la maquinaria empleada para transporte y mantenimiento (8).

Factor es un concepto genérico que engloba aquellas modificaciones del medio que, en mayor o menor medida, presentan unas características similares, pudiendo mencionar en este sentido la remoción de tierras como uno de los principales. No obstante, conviene recordar que nos podemos encontrar con otros, como el tránsito de maquinaria, que en determinadas circunstancias puede resultar muy agresivo. Para aclarar este concepto, es conve-

(8) En la mayoría de los casos, como se puede deducir, la afección se produce sobre todo en la fase de construcción, aunque la definición de una metodología completa y sistemática de EIArq debe tratar de contemplar un amplio abanico de proyectos que no tienen por qué pasar necesariamente por la realización inmediata de obras de construcción, como puede ser el caso de repoblaciones forestales o proyectos de ordenación territorial, o cuyos máximos efectos se producirían en la fase de funcionamiento, como embalses y pantanos. niente tener en cuenta que una EIArq siempre supone una previsión razonada, y que es tarea del arqueólogo determinar qué agentes y factores presentan una afección potencial sobre el entorno.

$\mathrm{Si}$ consideramos las acciones como aquellas actividades concretas que generan un efecto sobre el medio, y, consiguientemente, causa directa del impacto, encontramos englobadas dentro del factor remoción de tierras, y de mayor a menor grado de afección, algunos ejemplos como excavaciones, voladuras, préstamos, desbrozado, afirmado, rellenos, restituciones, etc... También, aunque en menor grado, se engloban en ese mismo factor distintas obras de fábrica y de mantenimiento como pueden ser desviaciones de cauce, drenajes e instalaciones menores.

A partir de esta propuesta de desagregación (9) de los componentes del proyecto se puede elaborar un esquema-modelo (Tab. 2), tomando como ejemplo la construcción de un Parque Eólico.

\begin{tabular}{|l|l|}
\hline Agente & $\begin{array}{l}\text { Aerogeneradores, canalizaciones para cableado y } \\
\text { red de tierra, subestación y centro de control, cen- } \\
\text { tros de transformación, accesos y viales internos. }\end{array}$ \\
\hline Factor & Remoción de tierras. \\
\hline Acción & $\begin{array}{l}\text { Excavación, voladura, préstamo, desbroce, afir- } \\
\text { mado, relleno, restitución, desviación de cauce, } \\
\text { drenaje. }\end{array}$ \\
\hline Agente & Parque de Maquinaria \\
\hline Factor & Tránsito de maquinaria \\
\hline Acción & Apisonado \\
\hline Agente & $\begin{array}{l}\text { Obras de fábrica y mantenimiento, instalaciones } \\
\text { menores }\end{array}$ \\
\hline Factor & Remoción de tierras \\
\hline Acción & Drenajes, desviaciones de cauce \\
\hline
\end{tabular}

Tab. 2. Análisis de los componentes de un proyecto de un Parque Eólico.

Por ámbito de afección del proyecto se entiende el territorio sobre el que un proyecto de obra actúa, para lo que hay que tener en cuenta tanto los datos referentes a la ubicación y extensión del mismo (sus características espaciales) como los provenientes de una descripción general de la zona. La experiencia de nuestro grupo en estudios de EIArq

(9) El término desagregación del proyecto está directamente tomado de Gómez, 1994. 
nos ha mostrado la conveniencia de diferenciar en los proyectos, de forma general, tres zonas:

1. La de afección propiamente dicha es sobre la que el proyecto incide de forma directa. Genéricamente, tomamos un área cuyo perímetro dista $50 \mathrm{~m}$. de las obras. La utilización indiscriminada de medios mecánicos masivos y la existencia de prácticas tales como la toma de préstamos, hace que las remociones de terrenos tiendan a superar, o al menos desbordar, los contornos previstos inicialmente.

2. La de incidencia, indirectamente afectada por el proyecto, y que abarca entre los 50 y los $200 \mathrm{~m}$. medidos desde el perímetro exterior de las obras. Este límite deriva de razones legales, dado el imperativo planteado por las Normas Subsidiarias $e$ Complementarias de Planeamento Urbanístico das Catro Provincias Galegas, según las cuales cualquier obra en el ámbito de los $200 \mathrm{~m}$. de cualquier yacimiento arqueológico debe ser informada.

3. Finalmente está la zona de muestreo, que abarca a partir de los $200 \mathrm{~m}$. y cuya consideración deriva de una razón metodológica, que viene dada por la conveniencia de reunir información arqueológica adicional para poder completar la valoración del PArq existente en el área de estudio, y fundar la EIArq en procedimientos e interpretaciones basados en el conocimiento del contexto de la zona y en estimaciones basadas en laArqueología del Paisaje. Por ello, los límites exteriores del área de muestreo pueden variar tanto en función de sus condiciones topográficas como del entorno arqueológico, oscilando generalmente entre 0,5 y $1 \mathrm{~km}$.

Una vez que el ámbito de afección del proyecto ha sido determinado, y las acciones susceptibles de generar impacto identificadas, podemos considerar que el proyecto ha sido convenientemente caracterizado. Será en una fase posterior (Evaluación del Impacto) cuando estemos en disposición de manejar los datos obtenidos.

\section{IDENTIFICACIÓN Y VALORACIÓN DE LOS BIENES AFECTADOS}

El proceso de identificación y valoración de los bienes arqueológicos y patrimoniales afectados por el proyecto debe iniciarse con los trabajos de campo necesarios para inventariar las entidades que componen el registro arqueológico existente en la zona y valorarlas. Se corresponde con la fase de inventario en los procesos de EIA. Sin embargo, el primer problema es relativo al objeto de trabajo (qué, cómo y para qué debe ser inventariado).

Aunque para enfocar la cuestión más adecuadamente sería necesario considerar los problemas específicos del registro del PArq gallego, un intento de sistematizar los procedimientos de valoración de los bienes afectados por un proyecto debe contemplar igualmente los problemas de índole general. Los objetos arqueológicos no son meros objetos, sino que "son valores intelectuales adscritos a elementos físicos que existenfuera de la sociedad pero no-son nada sin ella, ya que fueron el producto de otra sociedad distinta" (Criado, 1996a: 27).Así, el concepto de valor les es inherente, y es su consideración (su valoración) como documentos históricos lo que los convierte en objetos arqueológicos y en parte integrante del Registro Arqueológico. Por esta razón, de cara a su inclusión dentro de la EIArq, la valoración de los bienes debe adaptarse a un proceso especial, articulándose en tres fases: identificación, caracterización y valoración, cada una de las cuales responde a una necesidad concreta dentro del proceso, se orienta mediante unos criterios definidos y se articula a través de unos procedimientos específicos. Igualmente, este proceso de valoración debe organizarse como una operación secuencial que permitirá adjudicar un valor numérico (10) a cada bien inventariado, pues será este valor cuantitativo el que propicie su inclusión en la operación de evaluación del impacto.

\subsection{Fase de identificación}

En el inventario de objetos arqueológicos se puede establecer una distinción básica entre los elementos mejor definidos y todos aquellos puntos en los que han sido documentados indicios arqueológicos. Esto es, entre los objetos arqueológicos reales, constituidos por los yacimientos y elementos arqueológicos visibles en superficie y los objetos arqueológicos hipotéticos, constituidos por valoraciones e interpretaciones bien justificadas y documentadas sobre la posible existencia de yacimientos no evidenciados físicamente, es decir, caracterizados por su potencialidad.

Es imprescindible considerar los segundos porque una gran parte de los yacimientos arqueológi-

(10) Cuando hablamos de valor numérico nos estamos refiriendo a la inferencia para integrar, mediante parámetros cuantitativos, un determinado objeto dentro del proceso de evaluación. No nos referimos al valor "económico" del objeto. 
cos gallegos no se evidencian a simple vista y resulta difícil delimitar su presencia y extensión. Sin embargo, el estado actual de la investigación nos permite interpretar, categorizar y valorar hasta cierto punto esas evidencias potenciales (pudiendo así ser considerado el impacto potencial sobre las mismas antes de que sea efectivo). Para ello contamos con la posible recuperación de material arqueológico en la fase de prospección y, sobre todo, con modelos predictivos de localización de yacimientos, fundamentados en circunstancias como la existencia en el entorno de determinados tipos de yacimientos visibles, las características geográficas y topográficas de la zona, y analogías y extrapolaciones basadas en otros casos y zonas. La estrategia de trabajo de campo aplica para ello tres técnicas de prospección complementarias (extensiva, intensiva y de cobertura total).

El considerar no sólo el área directamente afectada por el proyecto, sino también el entorno, tiene como principal finalidad objetivar y formalizar nuestra propia percepción del espacio natural para que, ya en la fase de caracterización, esto pueda servir de base a un análisis formal del paisaje, en el que se incluyan tanto los componentes y unidades naturales derivados del análisis fisiográfico como aquellos componentes pertenecientes al espacio construido (11).

\subsection{Fase de clasificación}

La elaboración del inventario implica una clasificación de objetos arqueológicos, que permitirá establecer las pautas necesarias para modelar un sistema de valoración de los bienes arqueológicos y su inclusión dentro de un proceso global de EIArq (12). Para ello será preciso proceder a lacaracterización de los diferentes objetos que conforman el inventario. Esto supone definir los atributos o características particulares de cada objeto y, en última instancia, implica adscribirlo a una clase, entendiendo ésta como "el conjunto de objetos que responden a un patrón o 'molde' de apariencia y comportamiento determinado, y el proceso de clasificación como la adscripción de objetos a clases"

(11) Sobre el análisis formal como instrumento para la valoración de los elementos integrantes del paisaje véase Santos et alii, 1997.

(12) Para una comprensión más completa de nuestro sistema de inventario y clasificación véase Martínez, 1997 y Méndez, 1998.
(González et alii, 1997: 138). En definitiva, se trataría de transformar el mapa de puntos (entidades locacionales) en un mapa de yacimientos adscritos tipológica y crono-culturalmente (clasificados) (13). Como se podrá observar, el procedimiento de clasificación (y también el de identificación) implica una valoración arqueológica y patrimonial previa a la posterior fase de valoración de los bienes, por lo que conviene incidir en que estamos exponiendo las fases del proceso en una sucesión lógica (no cronológica), cuando en realidad estas fases interactúan constantemente.

El primer nivel de articulación del sistema clasificatorio propuesto estará constituido por una división en categorías (para evitar confusiones, denominamos así a las clases más genéricas), que sigue un orden jerárquico decreciente: paisajes arqueológicos, yacimientos, estructuras y materiales. La operación de clasificación debe comenzar por los objetos concretos, pues sólo a partir su conocimiento se puede ir más allá y articular los datos obtenidos en un contexto más amplio. Esta estrategia puede derivar de principios teóricos basados en la Arqueología del Paisaje ya que, en definitiva, se trata de "reconstruir e interpretar los paisajes arqueológicos a partir de los objetos que los concretan" (Criado, e.p.).

Así, de cara a la categorización de los objetos documentados (el problema fundamental suele estribar en cuándo una estructura o un conjunto de materiales se pueden calificar como yacimiento) habría que considerar una serie de parámetros de los que en primer lugar debemos señalar laimportancia y abundancia de la evidencia documental, entendiendo por ésta los conjuntos de materiales y/ o estructuras que testimonian la existencia de yacimientos arqueológicos. Evidentemente, no se trata de dos parámetros equiparables ya que el segundo posee un carácter cuantitativo y el primero un carácter cualitativo. Ambos deben ser simultáneamente estimados desde el momento en que pueden excluirse mutuamente, siendo el criterio del arqueólogo el que permite valorarlos tanto por sí mismos como en función de su relación con evidencias análogas en contextos similares.

Otro parámetro a tener en cuenta es el grado de estructuración de la evidencia. Para los restos documentados no visibles en superficie esto implica una disposición estratigráfica originaria, mientras

(13) Sobre la sistematización del registro de yacimientos arqueológicos véase Martínez, 1997. 
que para los yacimientos visibles, es su condición lo que nos revela el grado de estructuración, lo que no se debe confundir con la disposición o características de las estructuras que componen el yacimiento en caso de que las haya.

Por otra parte contamos con todos aquellos datos derivados de los modelos predictivos de localización de yacimientos, que pueden ser un factor más de apoyo para categorizar una evidencia como yacimiento. La aplicación razonada de estos modelos, en relación con labores de evaluación y corrección de impacto en obras públicas, ha permitido a nuestro grupo delimitar en numerosas ocasiones (a veces sin la concurrencia de evidencias materiales en superficie) zonas de potencial arqueológico posteriormente reveladas como emplazamientos arqueológicos reales; con más razón permitirá calificar un conjunto de evidencias como yacimiento en función de esos mismos factores. Concluimos así que otro de los parámetros a tener en cuenta a la hora de determinar cuándo una evidencia se puede considerar yacimiento es el entorno arqueológico.

Una vez efectuada la categorización, el procedimiento clasificatorio que proponemos nos llevaría a una distinción de rango entre las clases de objetos que conforman cada categoría. En primer lugar, si se conocen el uso y la función originarios del yacimiento, podemos establecer un primer rango (clases propiamente dichas), según se trate de áreas de asentamiento, lugares funerarios o cultuales, áreas de explotación económica, obras o edificios públicos o representaciones gráficas.

Dentro de cada una de esas clases, si se dispone de la información suficiente a partir de los restos materiales documentados durante la prospección, y/o si el yacimiento presenta unas características o atributos identificables que permiten su inclusión en una clase más concreta, es factible llevar a cabo una subdivisión. Para evitar problemas terminológicos, podemos referirnos a estas clases más concretas como tipos. Así, a una clase como lugar funerario, pueden pertenecer tipos diversos como necrópolis, túmulo, cista, fosa, urna, sepulcro o sarcófago. En el caso de los yacimientos de naturaleza invisible puede ser suficiente con determinar su clase, dada la dificultad intrínseca que presenta su proceso de caracterización, sobre todo si éste ha podido tener lugar durante la fase de prospección superficial.

Los criterios que regirán la posterior valoración de los bienes están determinados en buena medida a partir de las características genéricas de cada clase o tipo, por lo que dichas características, si bien sometidas a una constante revisión y ampliación derivada de la investigación básica en constante desarrollo, deben ser estandarizadas dentro de un margen de flexibilidad. Se trata, en definitiva, de crear un marco de referencia para el objeto arqueológico que pueda guiar y orientar al arqueólogo en la fase de valoración. Una clasificación tipológica de yacimientos no debe ser, a priori, tan problemática como la clasificación de las posibles estructuras identificables, tanto por la mayor diversidad tipológica existente entre éstas como por su carácter ambiguo, ya que pueden funcionar no sólo como entidades independientes, sino también, y sobre todo, como componentes de una unidad de articulación mayor, dentro de la categoría de yacimiento y/o de paisaje arqueológico. El mismo problema se presenta para el caso de los materiales documentados. El sistema de clasificación debe contemplar, por lo tanto, la posibilidad de considerar las estructuras como categorías agregadas, ligadas entre sí por relaciones de asociación y englobadas por relaciones de inclusión en una categoría de rango mayor.

En el nivel más concreto de los objetos, esto se plasma en el hecho de que cada pieza del registro de materiales puede ser agregada igualmente a un conjunto mayor (conjunto de materiales), que bien por sí misma o bien en asociación con otros conjuntos pueden (o no) ser caracterizadas y agregadas a una unidad mayor (que puede ser tanto una estructura aislada o agregada a un yacimiento, como el yacimiento por sí solo) y así sucesivamente hasta llegar a la unidad máxima, que en el caso concreto de una evaluación de impacto vendría definida directamente por la escala del proyecto.

Por último sistematizamos la clasificación en función de la adscripción cultural de los objetos, siempre que ésta haya podido ser determinada. Para paliar esta indeterminación, indisociable de la práctica arqueológica, proponemos establecer dos rangos dentro de la clasificación, el primero de los cuales presenta un carácter más genérico, lo que nos permite un mayor margen de flexibilidad en la adscripción (como puede ser el concepto de Neolítico). Cuando las características del objeto permiten una datación más ajustada, contamos con un segundo nivel de adscripción, más concreto y preciso (siguiendo con el ejemplo anterior, podríamos hablar de Neolítico Inicial, Neolítico Medio, Neolítico Final).

T. P., 56, n. ${ }^{\circ} 1,1999$ 


\subsection{Fase de valoración}

El proceso de valoración de los bienes afectados por un proyecto tiene como objetivo principal el poder adjudicar un valor cuantitativo a dichos bienes, de cara a su inclusión en el proceso global de evaluación. Este valor posee un carácter indicativo, ya que profundizar en cuestiones sobre qué es el valor arqueológico y cómo se puede gestionar desde el presente supondría salirse del horizonte de este trabajo (14). No obstante, debemos encauzar los distintos componentes valorativos desarrollados hasta el momento hacia nuestro objetivo. Estos componentes se plasman en una serie de entidades, dentro del sistema de información (15) en que nos manejamos, que se pueden calificar como valorativas y que, partiendo del inventario realizado, se articularía en dos ejes confluyentes. Por un lado, la situación patrimonial, como diagnóstico, se refiere esencialmente al estado de conservación del yacimiento en el presente (vestigio), mientras que la valoración arqueológica consiste en una estimación del valor arqueológico del yacimiento como instrumento para la reconstrucción histórica ( $\mathrm{sig}$ no), elaborada a partir de una síntesis de las evidencias documentadas, abarcando el entorno del yacimiento, y planteando una hipótesis sobre su significado dentro de aquel. Del cruce simultáneo de las dos entidades señaladas (valor como documento y estado de conservación) obtendríamos la valoración patrimonial del yacimiento, es decir, una serie de recomendaciones valoradas, tanto de cara al establecimiento de medidas correctoras como a las perspectivas de rentabilización social existentes, y que vendría a ser en definitiva el valor final o absoluto del bien. Tanto en el caso de la valoración arqueológica como en el de la situación patrimonial, creemos que es factible diseñar una estrategia de valoración que, partiendo de criterios flexibles, mantenga un cierto grado de homogeneidad en el resultado final. Estos son:

Significatividad: Consiste en el potencial informativo de los restos en función de su relación con el entorno y de las características que presenta como perteneciente a la clase o tipo en que haya sido incluido. Se trata del criterio básico de cara a una valoración arqueológica.

(14) Un tratamiento profundo de esta cuestión se puede encontrar en Ballart, 1997; Ballart et alii, 1996; Criado y González, 1994 y 1995, y González, 1995, e.p.

(15) La exposición detallada de la estructura organizativa de la base de datos SIA+ (referida en el texto) se encuentra en González, 1997
Consideramos que una primera valoración consiste precisamente en atribuir al objeto la condición de signo, dado que el objeto (sus atributos y su relación con el contexto) nos dice algo y lo calificamos por lo tanto como eje de una argumentación basada en el desciframiento de un código interpretativo. La perspectiva que nos interesa es aquella que el arqueólogo debe mantener a partir de un análisis de las formas en el espacio que se traduzca en la "aproximación a la racionalidad de unos modos de expresión social” (Velandia, 1994: 25).

Sin embargo, carece de sentido intentar delimitar, definir o sistematizar a priori las circunstancias que otorgan el significado a un objeto y/o a su entorno. Será el criterio subjetivo del arqueólogo el que estime el grado de significatividad que un objeto posee en función de:

- El número de atributos que pueden ser identificados como unidades de significado, articuladas entre niveles,progresivos que van desde el yacimiento como objeto con identidad propia hasta el último resto de cultura material, que aparecerá como objeto con identidad pero integrante de un contexto inmediato.

- Los indicios que permiten interrelacionarlo con otros componentes del entorno e introducirlo finalmente en una reconstrucción del paisaje arqueológico, valorando lo que éste, como última unidad estructural de significado, debe al objeto en cuanto componente del mismo.

Representatividad: Se trata de la relación que se establece entre las características o atributos propios del objeto y los atributos genéricos que presentan los de su clase y que, consiguientemente, han permitido su inclusión en la misma. Así, el objeto se convierte en representativo de una determinada clase o tipo por ser esas características y su relación con el contexto las típicas que presentan los objetos que la conforman. La valoración del grado de representatividad de un objeto se basaría por tanto en las mismas claves utilizadas para evaluar su significatividad, pero en este caso, una vez que se ha realizado la desagregación del objeto en unidades de significado, habrá que interrelacionar cada una de ellas con aquellas en las que se puede articular la clase a la que el objeto pertenece. Se trataría, en definitiva, de un criterio aplicable no tanto a la valoración arqueológica como a la situación patrimonial.

Excepcionalidad: Se establece, en el eje de la valoración arqueológica, en función de las anomalías que el yacimiento presenta en relación con las 
características genéricas de su clase. Si un objeto se considera representativo por poseer gran número de características típicas, lo será como excepcional cuando no sea así. Se trata de un criterio que, en este sentido, actúa en relación inversa al anterior, mientras que la estimación de un objeto como excepcional desde el punto de vista patrimonial tiene una doble lectura: puede ser coincidente con este punto de vista o bien puede valorar el grado de excepcionalidad de un yacimiento en la medida en que mantenga inusualmente bien conservados sus atributos.

Diversidad o Valor de Grupo: Depende directamente del entorno arqueológico del yacimiento, pudiendo hacerse una sintética distinción en cuatro niveles:

- Yacimientos aislados, donde debemos considerar tanto que distintos tipos presenten patrones de emplazamiento diferentes (16), como que la escala espacial se restrinja al entorno inmediato o abarque un área más amplia.

- Yacimientos del mismo tipo conformando conjuntos (i.e.: necrópolis tumulares, estaciones de grabados rupestres...).

- Yacimientos que conforman un paisaje sincrónico-cultural, en los que el yacimiento forma parte de un conjunto más amplio de yacimientos de distinta clase pero de un mismo horizonte cronocultural (i.e.: paisaje conformado por túmulos, grabados de cazoletas y asentamientos de época neolítica).

- Diversidad cronocultural de los yacimientos documentados en el área (17).

La concreción y definición de los criterios y procedimientos de la valoración del inventario permitirá su transformación en términos cuantitativos de cara al desarrollo del proceso de evaluación de impacto, y que dicha valoración sirva de fundamento para posteriores líneas de investigación relacionadas con la gestión integral de los recursos culturales, siguiendo la orientación de trabajos relacionados con la valorización social del patrimonio (Criado, 1996b).

Los criterios analizados hasta ahora (significa-

(16) Son especialmente significativos al respecto los trabajos de investigación y tesis de licenciatura de González Méndez, Méndez Fernández, Parcero Oubiña, Santos Estévez y Villoch Vázquez, así como la tesis doctoral en preparación de Méndez Fernández.

(17) La idea de relación de diversidad en función de la diacronía de un conjunto de yacimientos o de la articulación de un patrón productivo completo (que orienta la definición de paisaje sincrónico-cultural del punto anterior) se recoge en Zafra, 1996. tividad, representatividad, excepcionalidad y diversidad) orientan tanto la valoración arqueológica como el diagnóstico de la situación patrimonial. No obstante, en este punto es necesario transformar esos criterios de valoración en términos cuantitativos o parámetros de medida. Así, en cada uno de estos parámetros, y en lo que respecta a la valoración arqueológica, el índice puede adoptar unos valores de entre $l$ y 8 (la elección no es arbitraria, sino que se establece en función de la operación de evaluación de impacto que más adelante se desarrolla) siendo el arqueólogo el que, de forma ajustada y según los criterios expuestos y sus claves, los adjudique. Los parámetros de medida, a diferencia de los que rigen en la operación de evaluación del impacto, no están sometidos a un baremo objetivo, ya que no poseen una auténtica dimensión física. Recalcamos por esto que la fiabilidad del proceso depende no tanto de la rigidez de dichos parámetros como de la homogeneidad en la aplicación de los criterios de valoración. Como se puede observar en la tabla 3, los criterios de representatividad y excepcionalidad (en lo que respecta a la valoración arqueológica) son mutuamente excluyentes. Esto quiere decir que a la hora de operar con sus respectivos valores de cara a la obtención de un índice final, debe optarse por aquel de los dos que presenta un valor más alto. Así, el resultado final se deduce a partir de la elaboración de una media aritmética de los tres valores obtenidos, por lo que éste nunca podrá reflejar un índice superior a 8 .

Retomando el diagnóstico de la situación patrimonial, y a partir de los criterios reseñados anteriormente ( sin necesidad de cuantificación, puesto que serán otros los parámetros) procederíamos a efectuar un contraste entre su estado de conservación y su vulnerabilidad. Se trata por lo tanto de manejar una escala relativa en dos direcciones. Por un lado, consiste en calibrar el grado de conservación de un objeto en relación a todos aquellos similares de los que se tiene constancia. Por otro lado, aunque objetivamente dos objetos diferentes presenten un estado de conservación similar, el arqueólogo debe

\begin{tabular}{|l|c|}
\hline OBJETO & Valor Arqueológico \\
\hline Significatividad & $\mathrm{x}$ \\
\hline Representatividad & $\mathrm{y} 1$ \\
\hline Excepcionalidad & $\mathrm{y} 2$ \\
\hline Diversidad & $\mathrm{z}$ \\
\hline Valoración Arqueológica & $\frac{\mathrm{x}+\mathrm{y}+\mathrm{z}}{3}$ \\
\hline
\end{tabular}

Tab. 3. Índices de valoración arqueológica. 
discernir cuál de ellos posee un mayor grado de vulnerabilidad y, por lo tanto, debe valorar cuál muestra un mejor estado de conservación.

El resultado final del análisis de la situación patrimonial contempla cinco posibilidades, junto a las cuales hemos señalado el valor que adquieren como parámetros de cara a su inclusión en el proceso de valoración de los bienes: desaparecido $(\mathbf{0})$, casi destruido $(\mathbf{0 , 5})$, gravemente alterado $(\mathbf{1})$, poco alterado $(\mathbf{1 , 5})$ y no se aprecia alteración (2).

Como una adición de los valores obtenidos en las fases de valoración arqueológica y situación patrimonial, deducimos el valor final (valoración patrimonial) con el que el bien afectado figurará en las posteriores fases del proceso de evaluación, donde habrá de ser contrastado con otros índices referentes a los demás factores implicados. De ello se deduce que el nuevo índice oscilará entre un valor mínimo de $\mathbf{3}(3+0)$, en caso de que un objeto haya dejado de existir materialmente, y un máximo de $10(8+2)$, para aquellos objetos de suprema valía y condiciones excepcionales de conservación.

\section{EVALUACIÓN DEL IMPACTO}

Una vez realizados el análisis del proyecto y la valoración de los bienes afectados, cruzando entre sí los datos procedentes de ambas fases, es posible identificar los Impactos Arqueológicos concretos y valorarlos. Esta es la fase que, con propiedad, debemos denominar evaluación de impacto arqueológico. En ella se han considerar no sólo los objetos arqueológicos reales, sino también los objetos hipotéticos antes definidos, esto es: todos aquellos que, a pesar de no ser reconocidos empíricamente, fomentan una sospecha razonable de producir incidencias arqueológicas durante las obras.

El proceso de EIArq resulta más sencillo (en el plano operacional, no en el conceptual o interpretativo) cuando hay que tratar con objetos hipotéticos, constituidos en definitiva por valores intelectuales o interpretaciones, en cuyo caso la evaluación se tendrá que limitar a establecer previsiones razonadas sobre la presencia potencial de yacimientos no visibles superficialmente. Naturalmente, una vez que las obras hayan comenzado, en la medida en que las remociones del terreno permitan documentar información antes oculta, las previsiones de impacto pueden ser revisadas (si ya existían) o formuladas por vez primera. En cualquier caso, la evaluación del impacto sobre objetos arqueológicos hipotéticos siempre tendrá un carácter estimativo, a expensas de las incidencias que posteriormente revelen las labores de control y seguimiento arqueológicos, ya en fase de ejecución. Esto se relaciona directamente con la problemática específica que presentan los puntos y yacimientos que no son descubiertos hasta el momento en que se ven afectados por las obras, lo que concierne más a una metodología de control y corrección que a la de evaluación.

El proceso de evaluación permitirá prever el impacto en cuanto trate, fundamentalmente, con objetos arqueológicos reales, visibles en superficie. Es con este tipo de entidades con las que es factible aplicar criterios y procedimientos evaluativos completos. Los ejemplos de este tipo más destacados en Galicia son los monumentos tumulares de finalidad funeraria, que se documentan desde el Neolítico Final hasta finales de la Edad del Bronce; los castros, que constituyen asentamientos fortificados de la Edad del Hierro y los petroglifos.

\subsection{Inventario de impactos y criterios de evaluación de impacto}

La secuencia operacional de la evaluación partiría de la elaboración de un inventario de impactos, fase previa a la valoración de los mismos, que se plasmaría en la fórmula:una acción + una entidad arqueológica = un impacto. Así, del mismo modo que un acción puede presentar un impacto sobre más de un punto o yacimiento, se pueden dar circunstancias en las que más de una acción impacte sobre el mismo yacimiento (como puede ser el caso de un túmulo sobre el que haya circulado maquinaria y que haya servido para extraer tierras de préstamo).

Una vez inventariados los impactos, estaremos en disposición de entrar en la fase de evaluación. Esto requiere definir los criterios sobre los que se realiza, dentro de los cuales es posible establecer unos parámetros según el grado de afección de cada impacto concreto, y articular un procedimiento de realización. Así, es factible predefinir unas pautas operacionales que nos permitan ajustar nuestra propia percepción de la afección. Se trata, en definitiva, antes que de anular o suplir la limitación derivada del criterio subjetivo del arqueólogo, de canalizar éste hacia parámetros objetivables y de validez intersubjetiva. Los criterios son: extensiónmagnitud, incidencia y certidumbre.

Por extensión entendemos la superficie afectada por el impacto en relación a la estimada para el 
yacimiento y su entorno. La magnitud nos indica la relación proporcional entre el tipo de acción (por ejemplo, las perforaciones producidas para la instalación de un cierre) y la entidad arqueológica en sí (como pueden ser un castro o un túmulo), ya que las consecuencias de una misma acción no tienen por qué ser las mismas para distintos tipos de yacimientos, aunque objetivamente la extensión afectada sea la misma (sería parcial, por ejemplo, si afectase lateralmente al yacimiento). Por ello, la estimación de la magnitud se debe hacer simultáneamente a la de la extensión del impacto, siguiendo los mismos parámetros de medida; esto es: nula (0), puntual (1), parcial (2), amplia (4) y total (8). Siguiendo con el ejemplo, la instalación del cierre afectaría parcialmente al castro, pero su magnitud respecto a un túmulo sería amplia. Lógicamente, el incremento de la extensión afectada supone una mayor confluencia entre ambos parámetros.

Laincidencia hace referencia a la intensidad de la alteración producida y está directamente relacionada con el tipo de acción que genera el impacto.A diferencia del criterio extensión-magnitud, la determinación del criterio incidencia no se realiza en una sola dimensión, sino que la secuencia lógica de la operación se lleva a cabo en dos ámbitos diferentes: físico y visual, que deberán ser reflejados convenientemente a la hora de proceder a la valoración conjunta.

Así, dentro de la incidencia física, las pautas operacionales se corresponden con el análisis de aquellas acciones, más o menos profundas, englobadas dentro de factores como la remoción de tierras o el tránsito de maquinaria. La incidencia visual hace referencia a los proyectos que afectan a la estructura perceptual del paisaje, o lo que es lo mismo, a la legibilidad del mismo. En este sentido, resultan especialmente significativos los proyectos que implican edificación, aunque también se debe tener en cuenta en los estudios de evaluación de proyectos de ordenación territorial y repoblaciones forestales, que en Galicia poseen un alto grado de incidencia sobre los paisajes. En ambos casos, los parámetros hacen referencia a la intensidad de la alteración, bien por la profundidad de la misma en la incidencia física, bien por el grado de ocultación en la visual: nula (0), baja (1), media (2), alta (4) y total (8).

Huelga decir que si tanto la extensión-magnitud como la incidencia tienen valor 0 , estamos admitiendo que el proyecto no produce ningún tipo de afección. Por la misma razón, el criteriocertidumbre oscilará entre unos valores de $\mathbf{1}$ a 8 , y se vincula implícitamente a la evaluación en la fase de planificación del proyecto, ya que consiste en la certeza de que un agente determinado producirá un impacto sobre el objeto. En caso de que el impacto haya sido detectado en la fase de construcción, se trataría tanto de corroborar o modificar las previsiones efectuadas (si ya existían) como de reflejar la afección detectada si no estaba prevista (bien por suponer una acción no previsible sobre un yacimiento documentado, bien por tratarse de un punto o yacimiento no visible en superficie y sólo documentable durante las obras). Así, los parámetros a aplicar se relacionarán con las áreas de afección delimitadas previamente en función de las características del proyecto, y se puede valorar el impacto como poco probable (1), posible (2), probable (4) o seguro (8), ya que en este país y en lo que respecta a planificación no hay nada imposible.

\subsection{Caracterización del impacto}

El método de objetivación y cuantificación para la EIArq se orienta a la elaboración de una matriz de impactos en la que se reflejen los parámetros adoptados. Mediante la operación englobada en dicha matriz, la conjunción de los valores obtenidos en la evaluación con la valoración que se haya hecho de la propia entidad arqueológica se concretará en la definitiva caracterización del impacto.

El conducir el proceso de evaluación hasta este punto responde a la necesidad de someterlo a parámetros controlables, explícitos y objetivables. Ello supone que el resultado pueda ser reflejado en las categorías establecidas para las evaluaciones de impacto ambiental; es decir, impacto compatible, moderado, severo y crítico, y, consiguientemente, el objetivo del trabajo consistirá en la inclusión de un factor específico y singular, como es el PArq, dentro del proceso de EIA.

Uno de los aspectos del proceso de cuantificación que debe quedar mejor definido es el concerniente a la diferente función que desempeñan los criterios de evaluación en la operación, según la fase de realización del proyecto en la que ésta se efectúe. Así, hay que discernir entre evaluación en fase de planificación (estudio de evaluación), donde el impacto es potencial, y evaluación en fase de construcción (seguimiento y control), donde el impacto es efectivo.

T. P., 56, n. ${ }^{\circ} 1,1999$ 


\begin{tabular}{|c|c|c|c|c|c|c|}
\hline \multirow{2}{*}{ Extensión } & \multirow{2}{*}{ Magnitud } & \multicolumn{2}{|c|}{ Incidencia } & \multirow{2}{*}{ Certidumbre } & Valor YA & IMPACTO \\
\cline { 3 - 4 } & & Física & Visual & & \\
\hline $\mathrm{e}$ & $\mathrm{e}_{2}$ & $\mathrm{i}$ & $\mathrm{i}_{2}$ & $\mathrm{c}$ & $\mathrm{y}$ & $\frac{[(\mathrm{e}+\mathrm{i}) \cdot \mathrm{c}] \cdot \mathrm{y}}{10}$ \\
\hline
\end{tabular}

Tab. 4. Modelo propuesto para una Matriz de Evaluación de Impacto Arqueológico.

En el primer caso, es la estimación del valor del criterio de certidumbre el eje fundamental del estudio, mientras que la extensión y la incidencia deben ser consideradas de forma secundaria a la hora de valorar el tipo de impacto, por tratarse de criterios referentes a la caracterización de la afección que el proyecto presenta sobre la entidad arqueológica una vez que han comenzado las obras. Por el contrario, en las evaluaciones efectuadas en fase de seguimiento, la certidumbre puede adoptar un valor definitivo (en la medida en que la afección pasa de ser una estimación a ser una realidad) mientras que la valoración del impacto variará en función de la caracterización de la afección, efectuada tomando como referencia los criterios de extensión e incidencia.

El modelo de matriz de impactos que proponemos (Tab. 4) contempla una serie de campos para incluir los valores obtenidos en la medición de la extensión-magnitud, la incidencia y la certidumbre. Junto a estos valores, se incluye igualmente el valor final obtenido en la valoración de los bienes. De la operación realizada con ellos, reflejada en la matriz, se deduce el valor final del impacto (que oscilaría en una escala de valores de 1 a 128, siendo el impacto mayor cuanto mayor sea el índice obtenido).

Además de aumentar el grado de fiabilidad del proceso evaluativo, lo que permite el modelo de matriz propuesto, ya que se trata de una tabla individualizada para cada yacimiento o punto afectado, es reflejar todas aquellas acciones susceptibles de producir un impacto sobre dicho punto y permitir, consiguientemente, que sea el mismo procedimiento operacional el que nos indique cuál es la acción que presenta un mayor grado de afección.

\section{CONCLUSIONES}

Una vez valorados los impactos, se procede a estudiar las diferentes alternativas posibles para proponer una determinada estrategia de control y corrección del impacto, que presenta una problemática metodológica específica, sobrepasando el ámbito del presente texto (18). Hasta aquí hemos intentado exponer cómo se puede encardinar un proceso de EIArq dentro de una estrategia de sistematización de la gestión del patrimonio arqueológico. Hemos expuesto para ello un resumen de las bases metodológicas para estudios de Impacto Arqueológico y para incluir éstos dentro del proceso de EIA. Los criterios y procedimientos expuestos no son definitivos ni estáticos, sino que deben ser aplicados y contrastados en contextos distintos para establecer una metodología de evaluación que pueda llegar a ser utilizada por diferentes especialistas. Este mismo texto no es más que el boceto que resume la experiencia y el trabajo realizado al respecto y hasta el momento por nuestro Grupo de Investigación (19).

\section{BIBLIOGRAFÍA}

Amado, X.; Barreiro, D. y Martínez, M. ${ }^{\text {CC. }}$ (1998): "Evaluación y corrección de impacto arqueológico en obras públicas. Propuesta desde laArqueología del paisaje". Arqueología Espacial, 19-20: 153-64.

BALlART I HeRnÁNDEZ, J. (1997): El patrimonio histórico y arqueológico: valor y uso. Akal. Barcelona.

Ballart i Hernández, J.; Fullola i Pericot, J.M. ${ }^{a}$ y Petit i MendizÁbal, M.A. (1996): "El valor del patrimonio histórico". Complutum Extra, 6(II): 215-24.

Barreiro Martínez, D. y Villoch Vázquez, V. (1997): Un Modelo de Evaluación de Impacto Arqueológico: El Parque Eólico de Careón. CAPA, 1. Grupo de Investigación en Arqueología del Paisaje. Santiago

CONESA FERnÁNDEZ-Vítora, V. (1995): Guía metodológica para la Evaluación de Impacto Ambiental. Ediciones Mundi-Prensa. Bilbao.

Criado Boado, F. (1995): “El control arqueológico de obras de trazado lineal: planteamientos desde laArqueología del Paisaje". Actas del XXII CNA, I (Vigo, 1993): 2539. Consellería de Cultura / Concello de Vigo. Vigo.

- (1996a): "La Arqueología del futuro, ¿el Futuro de la Arqueología?". Trabajos de Prehistoria, 53(1): 15-36.

- (1996b): "Hacia un modelo integrado de investigación y gestión del Patrimonio Histórico: la cadena interpre-

(18) Sobre estrategias de corrección de impacto en fase de obra véase Amado et alii, 1998.

(19) Véase Barreiro y Villoch, 1997; Villoch y Barreiro, 1997; Criado et alii, e.p.a y Criado et alii e.p.b. 
tativa como propuesta". Boletín del Instituto Andaluz del Patrimonio Histórico, 16: 73-8.

- (e.p.): "La Arqueología del Paisaje como programa de gestión de recursos arqueológicos". En F. Criado (dir.) Memorias del Espacio. La culturización del espacio en la Prehistoria.Monografías de Arqueología del Paisaje, 1. Universidad de Santiago de Compostela. Santiago.

Criado Boado, F.; Amado Reino, J. y Martínez López, M. ${ }^{a}$ C. (1997). "Informe: Red de gasificación de Galicia. Corrección de impacto arqueológico". Revista de Arqueología, 198: 6-13.

Criado Boado, F. y González Méndez, M. (1994): “La puesta en valor del patrimonio arqueológico desde la perspectiva de la Arqueología del Paisaje". Conservación Arqueológica. Reflexión y debate sobre teoría y práctica. Cuadernos del Instituto Andaluz del Patrimonio Histórico, 2: 58-75.

- (1995): "La Socialización del Patrimonio Arqueológico desde la Perspectiva de la Arqueología del Paisaje". Actas del XXII CNA, I (Vigo, 1993): 261-6. Consellería de Cultura / Concello de Vigo. Vigo.

Criado Boado, F.; Méndez Fernández, F. y Villoch VázQUEZ, V. (e.p.a): "La Evaluación de ImpactoArqueológico como práctica arqueológica". En F. Criado (dir.): Memorias del Espacio. La culturización del espacio en la Prehistoria. Monografías deArqueología del Paisaje, 1. Universidad de Santiago de Compostela. Santiago.

Criado Boado, F.; Parcero Oubiña, C. Y Villoch VázQuez, V. (1995): "Control arqueológico del oleoducto Coruña-Vigo. Fase II: seguimiento de las obras de construcción”. Actas del XXII CNA, I (Vigo, 1993): 309-16. Consellería de Cultura / Concello de Vigo. Vigo.

Criado Boado, F.; Villoch Vázquez, V. y Barreiro MarTíNEZ, D. (e.p.b): Arqueología y parques Eólicos en Galicia 1: Proyecto marco de Evaluación de Impacto. $C A P A, 5$. Grupo de Investigación en Arqueología del Paisaje. Santiago.

Gómez OrEA, D. (1988): "Evaluación de Impacto Ambiental". Ciudad y territorio, 3: 5-32.

- (1994): Evaluación de Impacto Ambiental. Editorial Agrícola Española, S.A. Madrid.

GonzÁlez Alonso, J. (1989): Guías metodológicas para la elaboración de estudios de impacto ambiental. Vol. 2: Grandes presas. Ministerio de Obras Públicas y Transportes. Madrid.

GonZÁlez Méndez, M. (1995): “La concepción de un proyecto de valorización social del Patrimonio Arqueológico: el plan de Toques como referente". Archivo Español de Arqueología, 68: 225-41.

- (e.p.): "El vestigio como atracción del turismo, la interpretación como atracción del vestigio". III Jornadas de difusión del Patrimonio. Córdoba. 1996.

GonzÁlez Méndez, M.; Amado Reino, J. y Prieto MartíNEZ, P. (1995): “Control arqueológico de obras de trazado lineal: trabajos previos de la gasificación de Galicia”. Actas del XXII CNA, I (Vigo, 1993): 297-303. Consellería de Cultura / Concello de Vigo. Vigo.
GonZález Pérez, C.A. (1997): SIA+: Manual del Usuario. $C A P A, 3$. Grupo de Investigación en Arqueología del Paisaje. Santiago.

González Pérez, C.A.; del Río Pose, J.; Bóveda López, M. ${ }^{a}$ M. y CRIado Boado, F. (1997): "Tecnologías orientadas a objetos en la gestión de recursos culturales". Boletín del Instituto Andaluz del Patrimonio Histórico, 21: 137-45.

Martínez LóPeZ, M. ${ }^{a}$ C. (coord.) (1997): Contribución a un Sistema de Registro de Yacimientos Arqueológicos en Galicia. CAPA, 2. Grupo de Investigación en Arqueología del Paisaje. Santiago.

MÉndez Fernández, F. (1998): "Definición y análisis de poblados de la Edad del Bronce en Galicia”. En R. Fábregas (ed.): A Idade do Bronce en Galicia: novas perspectivas. Cadernos do Seminario de Sargadelos, 77: 183-90. Santiago.

Méndez Fernández, F.; González Méndez, M. y Amado Reino, J. (1995a): "Control arqueológico del oleoducto Coruña-Vigo. Fase I: trabajos previos y superficiales". Actas del XXII CNA, I (Vigo, 1993): 293-6. Consellería de Cultura / Concello de Vigo. Vigo.

Méndez Fernández, F.; Prieto Martínez, P. y Villoch VÁzQUEZ, V. (1995b): "Evaluación del impacto arqueológico de la autovías del M.O.P.T. (Lugo-A Coruña y Ourense-Porriño)". Actas del XXII CNA, I (Vigo, 1993): 305-8. Consellería de Cultura / Concello de Vigo. Vigo.

Ramos Millán, A.; Tapia Espinosa, A.; Aznar Pérez, J.C. y OSUnA VARGas, M.M.(1993): "El impacto arqueológico desde perspectivas conservacionistas. La autovía del Mediterráneo, Baza-Puerto Lumbreras. Tramo Cúllar-Velez Rubio (provincias de Granada y Almería)". Anuario de Arqueología Andaluza 1991, III: 169-82.

Santos Estévez, M.; Parcero Oubiña, C. y Criado Boado, F. (1997): "De la Arqueología Simbólica del Paisaje a la Arqueología de los Paisajes Sagrados". Trabajos de Prehistoria, 54(2): 61-80.

SuÁrez Cardona, F. (1989): Guías metodológicas para la elaboración de estudios de impacto ambiental. Vol. 1 : Infraestructuras lineales. Carreteras y ferrocarriles. Ministerio de Obras Públicas y Transportes. Madrid.

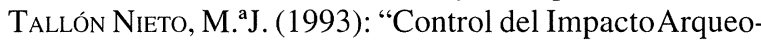
lógico de obras de iniciativa pública en Galicia”. Inventarios y Cartas Arqueológicas (Reunión de Soria, noviembre, 1991): 125-33. Junta de Castilla-León, Consejería de Cultura. Soria.

Velandia Jagua, C.A. (1994): SanAgustín.Arte, estructura y arqueología. Fondo de Promoción de la Cultura del Banco Popular. Bogotá.

Villoch Vázquez, V. y Barreiro Martínez, D. (1997): “AArqueología vs. Energía eólica?”. Boletín del Instituto Andaluz del Patrimonio Histórico, 20: 103-8.

ZAFRA DE LA TORRE, N.(1996): "Hacia una metodología para el estudio del Patrimonio Arqueológico". Complutum Extra, 6(II): 225-39.

T. P., 56, n. ${ }^{\circ} 1,1999$ 